\title{
Evolução do excesso de peso e fatores associados em mulheres de 10 a 49 anos em Pernambuco, Nordeste, Brasil ${ }^{1}$
}

\author{
Overweight changes and associated factors \\ in women aged 10 to 49 years from \\ Pernambuco, Brazilian Northeast
}

Emilia Chagas COSTA

Pedro Israel Cabral de LIRA ${ }^{3}$

Juliana Souza OLIVEIRA²

Risia Cristina Egito de MENEZES ${ }^{4}$

Fernanda Cristina de Lima Pinto TAVARES 3

Malaquias BATISTA FILHO ${ }^{5}$

\section{RE S U M O}

\section{Objetivo}

Este estudo tem como objetivo verificar a evolução do excesso do peso e fatores a ele associados, em mulheres de 10 a 49 anos, no Estado de Pernambuco, em pesquisas realizadas em 1997 e em 2006.

\section{Métodos}

Trata-se de estudo transversal, de base populacional, com amostra probabilística representativa dos meios urbano e rural de Pernambuco, abrangendo 1105 mulheres em 1997, e 1464 em 2006. Para mulheres entre 10 e 19 anos, foi utilizada a relação índice de massa corporal/idade, observados os seguintes parâmetros: déficit de peso: valores abaixo de -2 escores-Z; peso adequado: entre -2 e $<1$ escores-Z; sobrepeso: $\geq 1$ e $<2$ escores-Z; obesidade: $\geq 2$ escore-Z. Para mulheres com idade superior a 19 anos, consideraram-se os seguintes

1 Artigo elaborado a partir da tese de EC COSTA, intitulada "Anemia e excesso de peso: evolução e fatores associados em mulheres no período reprodutivo em Pernambuco". Universidade Federal de Pernambuco; 2013.

2 Universidade Federal de Pernambuco, Centro Acadêmico de Vitória, Núcleo de Educação Física. R. Alta do Reservatório, s/n., Bela Vista, 55608-680, Vitória de Santo Antão, PE, Brasil. Correspondência para/Correspondence to: EC COSTA. E-mail: <eccosta@hotmail.com>.

${ }^{3}$ Universidade Federal de Pernambuco, Centro de Ciências da Saúde, Departamento de Nutrição. Recife, PE, Brasil.

${ }^{4}$ Universidade Federal de Alagoas, Faculdade de Nutrição. Maceió, AL, Brasil.

${ }^{5}$ Universidade Federal de Pernambuco, Centro de Ciências da Saúde, Programa de Pós-Graduação em Nutrição. Recife, PE, Brasil.

Apoio: Fundo das Nações Unidas para a Infância, Ministério da Saúde, Instituto Nacional de Alimentação e Nutrição e Conselho Nacional de Desenvolvimento Científico e Tecnológico (Processos No. 505540/2004-5) e edital 30/2004. 
valores: déficit de peso: índice de massa corporal <18,5; normal: índice de massa corporal $\geq 18,5$ e $\leq 24,9$; sobrepeso: índice de massa corporal $\geq 25,0$ e $\leq 29,9$; obesidade: índice de massa corporal $\geq 30 \mathrm{~kg} / \mathrm{m}^{2}$. A análise de regressão de Poisson foi utilizada para avaliar a associação entre o excesso de peso e as variáveis explanatórias.

\section{Resultados}

Em relação ao estado nutricional no período compreendido entre 1997 e 2006, houve um aumento significativo no sobrepeso e obesidade, com estabilização na prevalência de déficit de peso. O excesso de peso aumentou de 32,8\% em 1997 para 41,7\% em 2006, representando um incremento de 27,1\%. As mulheres da Região Metropolitana do Recife, do Interior Urbano e Rural apresentaram um aumento de excesso de peso de 40,2\%, $6,8 \%$ e $45,2 \%$, respectivamente. Na avaliação dos fatores associados a esse agravo, em 2006, permaneceram significantes, após ajuste, baixa escolaridade (quatro ou menos anos de estudo), a posse de três ou mais bens de uso doméstico, idade e maior número de gestações, em contraste com o estudo de 1997, que apontava sobrepeso associado à idade e ao maior número de gestações.

\section{Conclusão}

O excesso de peso em mulheres em idade fértil, no Estado de Pernambuco, aumentou significativamente, observando-se menor ritmo no Interior Urbano. Evidencia-se nas categorias de condições socioeconômicas e microambientais mais favoráveis uma clara desaceleração no incremento temporal do excesso ponderal.

Termos de Indexação: Estudos transversais. Mulheres. Obesidade. Prevalência. Sobrepeso.

\section{A B S T R A C T}

\section{Objective}

To verify changes in overweight and associated factors in women aged 10 to 49 years from the state of Pernambuco in 1997 and 2006.

\section{Methods}

This population-based cross-sectional study used a probabilistic sample of women representative of the urban and rural areas of Pernambuco: 1,105 in 1997 and 1,464 in 2006. Body mass index-for-age was used for women aged 10 to 19 years; women with Z-score <-2 were considered underweight; women with -2 $\leq$ Z-score $<1$ were considered normal weight; women with $1 \leq Z$-score $<2$ were considered overweight; and women with $Z$-score $\leq 2$ were considered obese. Women aged more than 19 years were considered underweight when body mass index <18.5; normal weight when $18.5 \leq$ body mass index $\leq 24$.9; overweight when $25.0 \leq$ body mass index $\leq 29$.9; and obese when body mass index $\geq 30 \mathrm{~kg} / \mathrm{m}^{2}$. Poisson regression assessed the association between excess weight and the explanatory variables.

\section{Results}

In Pernambuco overweight and obesity rates increased significantly, and underweight rate remained stable between 1997 and 2006. Overweight increased from 32.8\% in 1997 to 41.7\% in 2006, an increase of $27.1 \%$. Women from the metropolitan region of Recife, and urban and rural areas of the state presented an increase in overweight of $40.2 \%, 6.8 \%$, and $45.2 \%$, respectively. The overweight-related factors that remained significant in 2006 after adjustment were: having three or more home appliances, low education level (four or fewer years of formal education), more pregnancies, and higher age, in contrast to the 1997 study where overweight was associated with having had more pregnancies and having higher age.

\section{Conclusion}

Excess body weight in women of childbearing age in the state of Pernambuco, increased significantly, with the lowest decreased seen in urban areas outside the state capital. In the categories of more favorable socioeconomic and microenvironment conditions, the increment of excess weight clearly slows down.

Indexing terms: Cross-sectional studies. Women. Obesity. Prevalence. Overweight.

\section{N T R O D U ÇÃ O}

No início do século passado, quando o sobrepeso e sua representação clínica mais ca- racterística - a obesidade -, eram eventos raros e percebidos como manifestação de bem-estar econômico, social, físico e até como idealização de valores estéticos nas artes plásticas, seria 
impensável admitir que o excesso de peso viesse a se converter na mais importante e desafiadora epidemia dos tempos atuais. O contraste dessas duas imagens históricas pode ser assumido como a ilustração marcante de um processo de mudanças sem precedentes na história nosográfica da humanidade: a transição epidemiológica nutricional' ${ }^{1}$.

Segundo estimativas da Organização Mundial da Saúde (OMS) ${ }^{2}$, pelo menos 2,8 milhões de pessoas morrem anualmente como resultado do excesso de peso, calculando-se que, em 2015, 2,3 bilhões de adultos serão obesos. Trata-se de um processo pandêmico que extrapola fronteiras geopolíticas, blocos econômicos, culturas e estratos sociais, com a observação surpreendente de que hoje, ao contrário do passado, são as camadas mais pobres dos países ricos ou de nações em desenvolvimento que constituem os segmentos mais expostos a sua ocorrência ${ }^{3}$.

Tipificada pelo acúmulo excessivo de tecido adiposo e tendo como fundamento etiopatogênico o elevado consumo de calorias ao lado de acentuada redução da atividade física que caracteriza o sedentarismo, a díade sobrepeso/ obesidade é a representação epidemiológica mais típica, no campo da saúde, do chamado estilo de vida ocidental, tendo como valores referenciais o consumismo e o conforto material, conhecido na antropologia política como "confortocracia". É uma situação que demarca os termos de uma "mutação antropológica"1. No processo saúde/ doença, o excesso de peso corporal aparece como a condição mais comum de um grupo de comorbidades que reúnem as dislipidemias, a doença vascular hipertensiva, o diabete melitus tipo 2 , alguns tipos de neoplasias, podendo eles ser reunidos sob a denominação genérica de Doenças Crônicas Não-Transmissíveis (DCNT). As DCNT representam, atualmente, o principal grupo causador de morte e incapacitação em escala mundial2,3, em que o Brasil alcança uma participação de $72 \%$ no obituário 4 . Tais doenças representam, ademais, o principal custo com a saúde, tanto na esfera pública quanto na privada, e a principal causa de incapacitações temporárias e definitivas.

A população adulta de mulheres constitui um grupo de elevada vulnerabilidade ao sobrepeso/obesidade, atingindo a prevalência de 49,0\% no Brasil, e de $35,0 \%$ em escala mundial ${ }^{2,5}$. Na Europa, no Mediterrâneo Oriental e na Região das Américas (classificação territorial da OMS), mais de $50,0 \%$ das mulheres adultas encontram-se nessa condição, caracterizando, de fato, uma pandemia ${ }^{2}$. No caso do Brasil, segundo quatro inquéritos nacionais realizados desde meados da década de 1970 até a Pesquisa de Orçamentos Familiares (POF, 2008/2009), as prevalências de excesso de peso aumentaram progressivamente de $28,7 \%$ para $48,0 \%$, enquanto a obesidade isoladamente elevou-se de $8,0 \%$ para $16,9 \%$. Entre as cinco macrorregiões, do País, o Nordeste Brasileiro registrou o maior crescimento comparativo do problema ${ }^{6}$.

Apesar das dificuldades conceituais e metodológicas em se avaliarem os determinantes do sobrepeso/obesidade, os estudos mais atuais concordam em destacar que os fatores socioambientais atuam de forma preponderante no desenvolvimento do problema ${ }^{2,7,8}$. Nas mulheres, a idade e paridade, como condições biológicas, assumem uma relação direta com o ganho excessivo de peso corporal, enquanto uma vertente dos determinantes socioeconômicos, com destaque para a renda e escolaridade, passaram a representar uma curiosa relação estatística, na medida em que as tendências atuais começam a indicar um aumento de peso nas faixas de menor renda e escolaridade ${ }^{8-11}$.

O interesse e até mesmo a prioridade de conhecer e acompanhar a evolução do excesso de peso e seus determinantes em mulheres em idade reprodutiva justifica-se na medida em que esse segmento populacional vem apresentando incrementos constantes na prevalência da DCNT. Ademais, no Estado de Pernambuco foram realizados três inquéritos de base populacional, representando tanto a população urbana quanto a rural, o que possibilitou o desenvolvimento deste 
trabalho, comparando as duas últimas pesquisas. O estudo teve como objetivo verificar a evolução do excesso do peso em mulheres de 10 a 49 anos, em 1997 e em 2006, e os fatores a ela associados, como subsidio para a tomada de medidas preventivas e de controle do problema no âmbito da Saúde Coletiva.

\section{MÉ TO D O S}

Trata-se de um estudo transversal, de base populacional, realizado a partir de dois inquéritos, demarcando um período de nove anos, com enfoque descritivo e analítico, mediante abordagem quantitativa. $\mathrm{O}$ artigo aqui apresentado teve como fonte primária os bancos de dados referentes à II e à III Pesquisa Estadual de Saúde e Nutrição (II e III PESN), realizadas nos anos de 1997 e 2006, com o objetivo de atualizar e ampliar o diagnóstico da situação de saúde, nutrição, alimentação e condições socioeconômicas da população materno-infantil do Estado de Pernambuco. Um novo inquérito com o mesmo conteúdo será efetuado em 2015, compondo um projeto de estudo de tendência secular nesse Estado.

A amostra, do tipo probabilística, por conglomerado, com representatividade para os estratos urbano e rural do Estado, foi constituída por mulheres em idade reprodutiva, na faixa etária de 10 a 49 anos. As duas amostras estudadas, com 1105 em 1997, e 1464 mulheres em 2006, têm um poder de $95 \%$ para detectar uma diferença observada de $8,9 \%$, admitindo um nível de significância de 5,0\%.

O trabalho de campo foi realizado por uma equipe de entrevistadores, antropometristas e técnicos encarregados da coleta e processamento dos exames laboratoriais. Os dados foram obtidos por meio de questionários e exames, realizados em unidades domiciliares, com registro das informações em formulários compostos por perguntas pré-codificadas, referentes a variáveis socioeconômicas, demográficas, antropométricas e laboratoriais das participantes. Os questionários preenchidos eram revisados diariamente para iden- tificação e correção oportuna de erros de preenchimento, checando-se, mediante novas visitas, as informações incompletas, duvidosas ou inconsistentes.

A presença de anemia foi avaliada em amostra de sangue venoso obtida por punção digital e analisada pelo equipamento HemoCue ${ }^{\circledR}$, de leitura imediata. A anemia foi definida segundo pontos de corte dos valores da hemoglobina estabelecidos pela Organização Mundial da Saúde ${ }^{12}$, sendo consideradas anêmicas as participantes com nível de hemoglobina inferior a $12 \mathrm{~g} / \mathrm{dL}$. A avaliação antropométrica foi realizada por antropometristas instruídos segundo procedimentos técnicos recomendados pela $\mathrm{OMS}^{13}$ e em observância às normas do Manual de Acompanhamento do Crescimento e Desenvolvimento do Ministério da Saúde ${ }^{14}$.

Na II Pesquisa Estadual de Saúde e Nutrição, o peso corporal foi obtido por meio de balança digital eletrônica Modelo Personal Line E-150/ Filizola ${ }^{\circledR}$, enquanto na III PESN utilizou-se balança digital Modelo MEA-03200/Plenna ${ }^{\circledR}$, ambas com capacidade máxima de 150 kg e precisão de $100 \mathrm{~g}$. Para aferição da estatura, nas II e III PESN, respectivamente, fez-se uso de estadiômetro portátil (Alturaexata ${ }^{\circledR}$, Ltda), com amplitude de $200 \mathrm{~cm}$ e precisão de $1 \mathrm{~mm}$ em toda a extensão. Para assegurar a acurácia das medidas, as aferições foram realizadas em duplicata para cada indivíduo, com a condição de que a diferença entre as avaliações não excedesse $0,5 \mathrm{~cm}$. Caso esse limite fosse ultrapassado, repetia-se a mensuração, anotando-se as duas medições com valores mais próximos e utilizando sua média para efeito de registro.

A relação peso/altura para mulheres entre 10 e 19 anos foi avaliada por meio do Índice de Massa Corporal (IMC)/ldade, a partir das recomendações da OMS ${ }^{15}$, considerando-se déficit: os valores abaixo de -2 escores-Z; adequado: entre -2 e $<1$ escores-Z; sobrepeso: $\geq 1$ escore-Z e $<2$ escore-Z; obesidade $\geq 2$ escore-Z. Para mulheres acima de 19 anos, na categorização do estado nutricional considerou-se IMC $<18,5 \mathrm{~kg} / \mathrm{m}^{2}$ como 
déficit de peso; como normal, IMC $\geq 18,5$ e $\leq 24,9$; como sobrepeso, IMC $\geq 25 \mathrm{~kg} / \mathrm{m}^{2}$; como obesidade $^{13}, \mathrm{IMC} \geq 30 \mathrm{~kg} / \mathrm{m}^{2}$.

As variáveis explanatórias foram representadas, de forma categórica, incluindo: situação geográfica do domicílio (Região Metropolitana do Recife, Interior Urbano e Interior Rural); renda familiar per capita $(<0,25 ; 0,25-0,49$ e $\geq 0,50$ salários-mínimos); escolaridade ( $<4 ; 4-7$ e $\geq 8$ em anos de estudo); posse de bens de consumo, representada em duas categorias (possuir três ou mais; e dois, um ou nenhum dos itens); número de pessoas residentes no domicílio $(<4 ; 4-7$ e $\geq 8$ pessoas); regime de ocupação da moradia (casa própria, alugada e outros); água de beber (tratada e outras formas); acesso à água (rede geral e outras formas); saneamento (rede geral, fossa com tampa e outras formas); destino do lixo (coletado e outras formas); idade (10-19, 20-29, $\geq 30$ ); número de gestações (nulíparas; 1-2; 3 ou mais), presença de anemia (anêmicas e não anêmicas); e cor da pele (parda/negra e branca).

Os dados das pesquisas foram digitados em dupla entrada, validados, processados e analisados utilizando-se o software Epi Info, versão 6.04. A avaliação antropométrica foi interpretada mediante o software Anthroplus-2007. Para as análises estatísticas, foram utilizados os programas Epi Info, versão 6.04, Statistical Package for the Social Sciences (SPSS) versão 12.0 e Stata 7.0.

A evolução temporal do excesso de peso foi determinada pela comparação das prevalências e seus intervalos de confiança para os anos de 1997 e 2006, e como razão de proporções para o ano de 1997. Para a comparação das variáveis categóricas intergrupos, utilizou-se o teste do Qui-quadrado (verificação de heterogeneidade) ou o teste do Qui-quadrado com correção de Yates para as variáveis dicotômicas, considerando-se como estatisticamente significante o valor de $p<0,05$.

As Razões de Prevalência (RP) para excesso de peso foram calculadas para cada variável de exposição por regressão de Poisson para os anos de 1997 e 2006. As variáveis com valor de $p<0,20$ nas análises bivariadas foram selecionadas para compor o modelo de regressão multivariado, adotando-se o modelo hierarquizado de entrada de variáveis, com a finalidade de avaliar o impacto das variáveis explanatórias. Esse procedimento foi realizado, em relação a 1997 e 2006, obedecendo-se a um processo de modelagem por blocos, de modo que inicialmente foram incluídas no bloco I as variáveis socioeconômicas, mantendo-se no modelo as variáveis que evidenciaram associação significante com o excesso de peso $(p<0,05)$, No segundo bloco, foram introduzidas as variáveis demográficas e biológicas como permanência das variáveis com valor de $p<0,05$, sendo ajustadas pelas demais. Os resultados foram expressos por RP ajustadas com respectivos Intervalos de Confiança de 95\% (IC95\%), sendo considerados estatisticamente significantes valores $p<0,05$.

Os dois projetos de pesquisa utilizados no estudo foram aprovados pelos respectivos Comitês de Ética em Pesquisa em Seres Humanos do Centro Ciências da Saúde da Universidade Federal de Pernambuco (II PESN - 27/02/97) e do Instituto de Medicina Integral Prof. Fernando Figueira (III PESN - 09/11/05), seguindo a Resolução n 196/96 do Conselho Nacional de Saúde. Assim, as mulheres que aceitaram participar da pesquisa assinaram o termo de consentimento livre e esclarecido e foram submetidas às entrevistas, exames antropométricos e bioquímicos. Os casos com diagnóstico de distúrbio nutricional ou algum outro problema de saúde foram encaminhados ao serviço de saúde mais acessível do município, para o devido acompanhamento.

\section{RESULTADOS}

No gráfico anexo apresentam-se as prevalências do estado nutricional nos anos de 1997 e 2006, verificando-se que o aumento da obesidade proporcionalmente foi bem maior que a elevação do sobrepeso, enquanto o déficit de peso se manteve no mesmo patamar de ocorrência. 


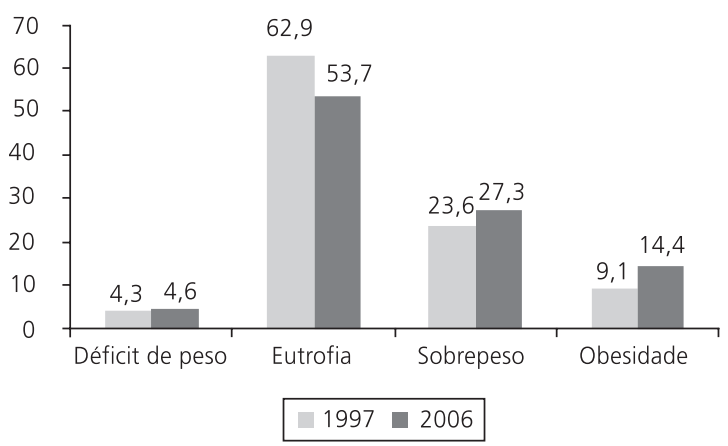

Figura 1. Evolução do estado nutricional de mulheres de 10 a 49 anos, segundo o índice de massa corporal. Pernambuco, nos anos 1997 e 2006.

Na Tabela 1 apresentam-se os resultados referentes à estratificação intragrupo das variáveis explanatórias e as comparações intergrupos representadas pelas prevalências encontradas em 1997 e 2006. No primeiro enfoque, demonstra-se que, no inquérito de 1997, a área geográfica, os bens de consumo e o número de pessoas por domicílio atuaram como fatores de variação estatisticamente significante, enquanto a coleta de lixo e o regime de ocupação de moradia (casa própria) apontaram valores de " $p$ " bem próximos dos níveis de discriminação de diferenças significativas. Desse modo, as maiores prevalências de excesso de peso foram encontradas entre as mulheres que residiam no interior urbano, que possuíam três ou mais bens de consumo e que apresentavam de quatro a sete pessoas por domicílio.

Já no III Inquérito, na maioria do agrupamento de variáveis analisadas, as diferenças não foram estatisticamente significativas, com exceção dos anos de estudo e dos bens de consumo. Os anos de escolaridade formal, analisados em três categorias, não apresentaram associação estatística com a ocorrência de excesso de peso em 1997, enquanto em 2006 o grupo com 0 a 4 anos de estudo apresentou maior prevalência de excesso de peso $(51,1 \%)$ do que o grupo com oito ou mais anos de estudo (37,2\%). No segundo plano de análise, evidenciou-se que, em todas as categorias comparadas, aumentou a prevalência de excesso de peso corporal, principalmente no interior rural, na faixa de renda mais baixa ( $\leq$ salário-mínimo per capita), com escolaridade igual ou inferior a quatro anos (elevação de 63,2\%), nas mulheres que habitavam residências alugadas ou outro tipo de ocupação de moradia, nas residências não servidas por rede de água tratada e nos domicílios sem acesso à coleta de lixo (Tabela1).

Como se observa na Tabela 2, nos dois anos avaliados, a idade das mulheres se correlacionou fortemente com a frequência do excesso de peso $(p<0,0001)$, que se elevou de $15,2 \%$ para $52,2 \%$ (1997), e de $21,5 \%$ para $56,6 \%$ (2006), quando comparadas as mulheres com 19 anos ou menos, com aquelas de 30 anos ou mais. Também o histórico reprodutivo (nulíparas e paridade de um, dois e três ou mais nascimentos) se correlacionou significativamente com a frequência do excesso de peso corporal. A ocorrência de anemia e a cor da pele não figuraram como condições de diferenças estatisticamente significativas, observando-se que a classificação racial não foi notificada no inquérito de 1997. Em todas as categorias examinadas houve aumento de ocorrência de excesso de peso, destacando-se o grupo de menores de 19 anos (elevação de 41,4\%) e nulíparas (elevação de 37,3\%)

$\mathrm{Na}$ análise de regressão de Poisson, mantiveram-se no modelo final entre as variáveis socioeconômicas: a posse de três ou mais bens de uso doméstico e a baixa escolaridade (quatro ou menos anos de estudo) no ano de 2006, ao lado das variáveis biológicas, nos anos de 1997 e 2006, com maiores razões de prevalências relacionadas ao aumento da idade e ao número de gestações (Tabela 3).

\section{DIS C U S S Ã O}

São de fato surpreendentes as mudanças produzidas no processo saúde/doença, caracterizando o que se convencionou chamar, a partir 
Tabela 1. Excesso de peso em mulheres de 10 a 49 anos, segundo a localização geográfica do domicílio, condições socioeconômicas, regime de ocupação e saneamento domiciliar no Estado de Pernambuco, nos anos 1997 e 2006.

\begin{tabular}{|c|c|c|c|c|c|c|c|c|c|}
\hline \multirow{3}{*}{ Variáveis } & \multicolumn{8}{|c|}{ Excesso de Peso } & \multirow{3}{*}{$\begin{array}{c}\text { Aumento } \\
\text { 1997/2006 } \\
\%\end{array}$} \\
\hline & \multicolumn{4}{|c|}{ PESN-1997 } & \multicolumn{4}{|c|}{ PESN-2006 } & \\
\hline & $\mathrm{N}$ & $\mathrm{n}$ & $\%$ & IC96\% & $\mathrm{N}$ & $\mathrm{n}$ & $\%$ & IC96\% & \\
\hline \multicolumn{10}{|c|}{ Situação geográfica do domicílio } \\
\hline Estado & 1105 & 362 & 32,8 & $30,0-35,6$ & 1464 & 610 & 41,7 & $39,1-44,2$ & $27,1^{\mathrm{c}}$ \\
\hline RMR & 415 & 125 & 30,1 & $25,8-34,8$ & 415 & 175 & 42,2 & $37,4-47,0$ & $40,2^{c}$ \\
\hline Interior Urbano & 363 & 145 & 39,9 & $34,9-45,2$ & 366 & 156 & 42,6 & $37,5-47,8$ & 6,8 \\
\hline Interior Rural & 327 & 92 & 28,1 & $\begin{array}{c}23,4-33,4 \\
p=0,002\end{array}$ & 683 & 279 & 40,8 & $\begin{array}{c}37,1-44,6 \\
p=0,8\end{array}$ & $45,2^{c}$ \\
\hline \multicolumn{10}{|c|}{ Renda familiar per capita, SM } \\
\hline $\begin{array}{l}<0,25 \\
0,25-0,49 \\
\geq 0,50\end{array}$ & $\begin{array}{l}351 \\
303 \\
451\end{array}$ & $\begin{array}{l}102 \\
100 \\
160\end{array}$ & $\begin{array}{l}29,1 \\
33,0 \\
35,5\end{array}$ & $\begin{array}{c}24,4-34,2 \\
27,8-38,6 \\
31,1-40,1 \\
p=0,2\end{array}$ & $\begin{array}{l}714 \\
460 \\
290\end{array}$ & $\begin{array}{l}305 \\
190 \\
115\end{array}$ & $\begin{array}{l}42,7 \\
41,3 \\
39,7\end{array}$ & $\begin{array}{c}39,1-46,4 \\
36,8-46,0 \\
34,0-45,6 \\
p=0,7\end{array}$ & $\begin{array}{l}46,7^{\mathrm{c}} \\
25,1^{\mathrm{a}} \\
11,8\end{array}$ \\
\hline \multicolumn{10}{|l|}{ Anos de estudo } \\
\hline $\begin{array}{l}0-3 \\
4-7 \\
\geq 8\end{array}$ & $\begin{array}{l}463 \\
380 \\
262\end{array}$ & $\begin{array}{c}145 \\
128 \\
89\end{array}$ & $\begin{array}{l}31,3 \\
33,7 \\
34,0\end{array}$ & $\begin{array}{c}27,1-35,8 \\
29,0-38,7 \\
28,3-40,1 \\
p=0,7\end{array}$ & $\begin{array}{l}354 \\
604 \\
506\end{array}$ & $\begin{array}{l}181 \\
241 \\
188\end{array}$ & $\begin{array}{l}51,1 \\
39,9 \\
37,2\end{array}$ & $\begin{array}{l}45,8-56,4 \\
36,0-43,9 \\
32,9-41,5 \\
p<0,001\end{array}$ & $\begin{array}{c}63,3^{c} \\
18,4 \\
9,4\end{array}$ \\
\hline \multicolumn{10}{|l|}{ Bens de consumo* } \\
\hline $\begin{array}{l}\text { Possuir três ou mais } \\
\text { Possuir dois/um/nenhum }\end{array}$ & $\begin{array}{l}845 \\
260\end{array}$ & $\begin{array}{c}292 \\
70\end{array}$ & $\begin{array}{l}34,6 \\
26,9\end{array}$ & $\begin{array}{c}31,4-37,9 \\
21,7-32,8 \\
p=0,02\end{array}$ & $\begin{array}{c}1228 \\
236\end{array}$ & $\begin{array}{c}533 \\
77\end{array}$ & $\begin{array}{l}43,4 \\
32,6\end{array}$ & $\begin{array}{c}40,6-46,2 \\
26,8-39,1 \\
p=0,002\end{array}$ & $\begin{array}{l}25,4^{c} \\
21,2\end{array}$ \\
\hline \multicolumn{10}{|l|}{ Pessoas/domicílio (n) } \\
\hline $\begin{array}{l}<4 \\
4-7 \\
\geq 8\end{array}$ & $\begin{array}{l}112 \\
625 \\
368\end{array}$ & $\begin{array}{c}36 \\
223 \\
103\end{array}$ & $\begin{array}{l}32,1 \\
35,7 \\
28,0\end{array}$ & $\begin{array}{c}23,8-41,7 \\
31,9-39-6 \\
23,5-32,9 \\
p=0,04\end{array}$ & $\begin{array}{l}623 \\
590 \\
251\end{array}$ & $\begin{array}{c}258 \\
256 \\
96\end{array}$ & $\begin{array}{l}41,4 \\
43,4 \\
38,2\end{array}$ & $\begin{array}{c}37,5-45,4 \\
39,4-47,5 \\
32,3-44,6 \\
p=0,4\end{array}$ & $\begin{array}{l}29,0 \\
21,6^{\mathrm{a}} \\
36,4^{\mathrm{b}}\end{array}$ \\
\hline \multicolumn{10}{|c|}{ Regime de ocupação da moradia } \\
\hline $\begin{array}{l}\text { Própria } \\
\text { Alugada } \\
\text { Outros }\end{array}$ & $\begin{array}{l}694 \\
205 \\
206\end{array}$ & $\begin{array}{c}244 \\
60 \\
58\end{array}$ & $\begin{array}{l}35,2 \\
29,3 \\
28,2\end{array}$ & $\begin{array}{c}31,6-38,8 \\
23,2-36,1 \\
22,2-34,9 \\
p=0,08\end{array}$ & $\begin{array}{c}1005 \\
168 \\
291\end{array}$ & $\begin{array}{c}415 \\
71 \\
124\end{array}$ & $\begin{array}{l}41,3 \\
42,3 \\
42,6\end{array}$ & $\begin{array}{c}38,2-44,1 \\
34,8-50,1 \\
36,9-48,5 \\
p=0,4\end{array}$ & $\begin{array}{l}17,3^{\mathbf{b}} \\
44,4^{\mathbf{b}} \\
51,1^{\mathbf{b}}\end{array}$ \\
\hline \multicolumn{10}{|l|}{ Abastecimento de água } \\
\hline $\begin{array}{l}\text { Rede geral } \\
\text { Outras formas }\end{array}$ & $\begin{array}{l}748 \\
357\end{array}$ & $\begin{array}{l}254 \\
108\end{array}$ & $\begin{array}{l}34,0 \\
30,3\end{array}$ & $\begin{array}{c}30,6-37,5 \\
25,6-35,3 \\
p=0,2\end{array}$ & $\begin{array}{l}855 \\
609\end{array}$ & $\begin{array}{l}362 \\
248\end{array}$ & $\begin{array}{l}42,3 \\
40,7\end{array}$ & $\begin{array}{c}39,0-45,7 \\
36,8-44,7 \\
p=0,5\end{array}$ & $\begin{array}{l}24,4^{c} \\
34,3^{c}\end{array}$ \\
\hline \multicolumn{10}{|l|}{ Água de beber } \\
\hline $\begin{array}{l}\text { Tratada } \\
\text { Outras formas }\end{array}$ & $\begin{array}{l}640 \\
465\end{array}$ & $\begin{array}{l}219 \\
143\end{array}$ & $\begin{array}{l}34,2 \\
30,8\end{array}$ & $\begin{array}{c}30,6-38,0 \\
26,6-35,2 \\
p=0,2\end{array}$ & $\begin{array}{l}774 \\
690\end{array}$ & $\begin{array}{l}331 \\
279\end{array}$ & $\begin{array}{l}42,8 \\
40,4\end{array}$ & $\begin{array}{c}39,2-46,3 \\
36,8-44,2 \\
p=0,4\end{array}$ & $\begin{array}{l}25,1^{c} \\
31,2^{c}\end{array}$ \\
\hline \multicolumn{10}{|l|}{ Saneamento } \\
\hline $\begin{array}{l}\text { Rede geral } \\
\text { Fossa com tampa } \\
\text { Outras formas }\end{array}$ & $\begin{array}{l}295 \\
400 \\
410\end{array}$ & $\begin{array}{l}109 \\
131 \\
122\end{array}$ & $\begin{array}{l}36,9 \\
32,8 \\
29,8\end{array}$ & $\begin{array}{c}31,5-42,8 \\
28,2-37,6 \\
25,4-34,7 \\
p=0,1\end{array}$ & $\begin{array}{l}513 \\
497 \\
454\end{array}$ & $\begin{array}{l}227 \\
204 \\
179\end{array}$ & $\begin{array}{l}44,2 \\
41,0 \\
39,4\end{array}$ & $\begin{array}{c}39,1-48,7 \\
36,7-45,5 \\
34,9-44,1 \\
p=0,3\end{array}$ & $\begin{array}{l}19,8^{\mathrm{a}} \\
25,0^{\mathbf{b}} \\
32,2^{\mathrm{b}}\end{array}$ \\
\hline \multicolumn{10}{|l|}{ Destino do lixo } \\
\hline $\begin{array}{l}\text { Coletado } \\
\text { Outras formas }\end{array}$ & $\begin{array}{l}582 \\
523\end{array}$ & $\begin{array}{l}204 \\
158\end{array}$ & $\begin{array}{l}35,1 \\
30,2\end{array}$ & $\begin{array}{c}31,2-39,1 \\
26,3-34,4 \\
p=0,09\end{array}$ & $\begin{array}{l}889 \\
575\end{array}$ & $\begin{array}{l}378 \\
232\end{array}$ & $\begin{array}{l}42,5 \\
40,3\end{array}$ & $\begin{array}{c}39,2-45,8 \\
36,3-44,5 \\
p=0,4\end{array}$ & $\begin{array}{l}21,1^{\mathbf{b}} \\
33,4^{\mathrm{c}}\end{array}$ \\
\hline
\end{tabular}

Nota: "televisão, geladeira, fogão e rádio; Nível de significância: ${ }^{a} p<0,05 ;{ }^{b} p<0,01 ;{ }^{c} p<0,001$ (diferenças entre os anos).

PESN: Pesquisa Estadual de Saúde e Nutrição; RMR: Região Metropolitana de Recife; SM: Salário-Mínimo; IC95\%: Intervalo de Confiança de $95 \%$. 
Tabela 2. Excesso de peso em mulheres de 10 a 49 anos, segundo a idade, condições reprodutivas, anemia e raça. Pernambuco, 1997 e 2006.

\begin{tabular}{|c|c|c|c|c|c|c|c|c|c|}
\hline \multirow{3}{*}{ Variáveis } & \multicolumn{8}{|c|}{ Excesso de Peso } & \multirow{3}{*}{$\begin{array}{c}\text { Aumento } \\
1997 / 2006 \\
\%\end{array}$} \\
\hline & \multicolumn{4}{|c|}{ PESN-1997 } & \multicolumn{4}{|c|}{ PESN-2006 } & \\
\hline & $\mathrm{N}$ & $n$ & $\%$ & IC96\% & $\mathrm{N}$ & $n$ & $\%$ & IC96\% & \\
\hline \multicolumn{10}{|l|}{ Idade (anos) } \\
\hline $10-19$ & 303 & 46 & 15,2 & $11,4-19,8$ & 297 & 64 & 21,5 & $17,1-26,7$ & 41,4 \\
\hline $20-29$ & 465 & 140 & 30,1 & $26,01-34,5$ & 642 & 249 & 38,8 & $35,0-42,7$ & $28,9^{b}$ \\
\hline \multirow[t]{2}{*}{$\geq 30$} & 337 & 176 & 52,2 & $46,7-57,6$ & 525 & 297 & 56,6 & $52,2-60,8$ & 8,4 \\
\hline & & & & $p<0,0001$ & & & & $p<0,0001$ & \\
\hline \multicolumn{10}{|c|}{ Número de gestações } \\
\hline Nulíparas & 283 & 47 & 16,6 & $12,6-21,6$ & 250 & 57 & 22,8 & $17,8-28,6$ & 37,3 \\
\hline $1-2$ & 446 & 146 & 32,7 & $28,4-37,3$ & 654 & 244 & 37,3 & $33,6-41,1$ & 14,1 \\
\hline 3 ou mais & 376 & 169 & 44,9 & $39,8-50,2$ & 560 & 309 & 55,2 & $50,9-59,3$ & $22,9^{b}$ \\
\hline & & & & $p<0,0001$ & & & & $p<0,0001$ & \\
\hline \multicolumn{10}{|l|}{ Anemia } \\
\hline Anêmicas & 207 & 63 & 30,4 & $24,3-37,3$ & 236 & 88 & 37,3 & $31,2-43,8$ & 22,7 \\
\hline Não anêmicas & 898 & 291 & 33,3 & $\begin{array}{c}29,4-35,6 \\
p=0,4\end{array}$ & 1228 & 522 & 42,5 & $\begin{array}{c}39,7-45,3 \\
p=0,1\end{array}$ & $27,6^{c}$ \\
\hline \multicolumn{10}{|l|}{ Raça } \\
\hline Parda/Negra & - & - & - & - & 1056 & 452 & 42,8 & $39,8-45,8$ & - \\
\hline Branca & - & - & - & - & 408 & 158 & 38,7 & $\begin{array}{c}34,0-43,7 \\
p=0,2\end{array}$ & - \\
\hline
\end{tabular}

Nota: Nível de significância: ${ }^{a} p<0,05 ;{ }^{b} p<0,01 ;{ }^{c} p<0,001$ (diferenças entre os anos).

PESN: Pesquisa Estadual de Saúde e Nutrição; IC95\%: Intervalo de Confiança de 95\%.

dos anos 1960, de transição epidemiológica. Esse processo apresentou, no campo da nutrição, sua configuração mais peculiar, tipificada na substituição das carências nutricionais globais e específicas pela emergência epidêmica (ou pandêmica) do sobrepeso/obesidade. Por sua natureza, magnitude e consequências, representa um tema desafiador para os gestores públicos da saúde e para a sociedade, na medida em que altera radicalmente o quadro de prioridades da área. Faz-se necessária a mobilização de estratégias criativas para responder ao cenário epidemiológico que está emergindo, o que demanda ações ainda não validadas pela rotina dos programas.

Em nível nacional, em um período de cerca de 35 anos, a tendência secular do estado nutricional da população brasileira apresentou aumento expressivo tanto do excesso de peso quanto da obesidade em ambos os sexos, enquanto também se reduziu o déficit de peso na relação peso/ altura $^{6}$. O ritmo de crescimento da obesidade no Brasil, se mantido nos próximos 10 anos, produzirá uma situação semelhante à dos Estados Unidos, onde o problema atinge $31,8 \%$ da população adulta $^{2}$

No caso de Pernambuco, no espaço de apenas nove anos, a prevalência do excesso de peso em mulheres em idade reprodutiva aumentou $27 \%$, o que significa, como tendência linear, uma taxa anual de $3 \%$. Inesperadamente, foi a zona rural, historicamente a mais atingida pelas carências nutricionais, o espaço geográfico mais comprometido. É pertinente observar que a elevação da prevalência de sobrepeso/obesidade em mulheres em idade fértil tem ocorrido num ritmo bem mais elevado - praticamente duas vezes mais -, que a redução do déficit de estatura infantil, aqui retratado como situação de natureza oposta ao excesso de peso. Essa diferença no ritmo de mudanças dos dois problemas é o aspecto 
Tabela 3. Regressão de Poisson para excesso de peso em mulheres de 10 a 49 anos segundo condições socioeconômicas, idade e número de gestações. Pernambuco, 1997 e 2006.

\begin{tabular}{|c|c|c|c|c|c|c|}
\hline \multirow[t]{2}{*}{ Variáveis } & \multicolumn{3}{|c|}{ RP não ajustada } & \multicolumn{3}{|c|}{ RP ajustada } \\
\hline & $\mathrm{RP}$ & IC95\% & $p$ & $\mathrm{RP}$ & IC95\% & $p$ \\
\hline \multicolumn{7}{|l|}{$1997^{a}$} \\
\hline \multicolumn{7}{|l|}{ Bloco I } \\
\hline \multicolumn{7}{|l|}{$N^{\circ}$ de pessoas/domicilio } \\
\hline$\geq 8$ & 1 & & & 1 & & \\
\hline $4-8$ & 1,27 & $1,00-1,61$ & 0,04 & 1,25 & $0,99-1,58$ & 0,06 \\
\hline$<4$ & 1,14 & $0,78-1,68$ & 0,5 & 1,15 & $0,79-1,68$ & 0,47 \\
\hline \multicolumn{7}{|l|}{ Bloco II } \\
\hline \multicolumn{7}{|l|}{ Idade (anos) } \\
\hline 10-19 & 1 & & & 1 & & \\
\hline $20-29$ & 1,98 & $1,42-2,77$ & 0,0001 & 1,51 & $1,01-2,24$ & 0,04 \\
\hline$\geq 30$ & 3,44 & $2,49-4,76$ & 0,0001 & 2,53 & $1,69-3,80$ & 0,0001 \\
\hline \multicolumn{7}{|l|}{$N^{\circ}$ de gestações } \\
\hline Nulíparas & 1 & & & 1 & & 0,1 \\
\hline $1-2$ & 1,97 & $1,42-2,74$ & 0,0001 & 1,38 & $0,94-2,06$ & 0,02 \\
\hline 3 ou mais & 2,70 & $1,96-3,74$ & 0,0001 & 1,59 & $1,07-2,38$ & $2006^{b}$ \\
\hline \multicolumn{7}{|l|}{$2006^{b}$} \\
\hline \multicolumn{7}{|l|}{ Bloco I } \\
\hline \multicolumn{7}{|l|}{ Anos de estudo } \\
\hline $0-3$ & 1,32 & $1,11-1,57$ & 0,002 & 1,39 & $1,16-1,67$ & 0,001 \\
\hline$\geq 4$ & 1 & & & 1 & & \\
\hline \multicolumn{7}{|l|}{ Bens de consumo* } \\
\hline Possuir três/mais & 1,33 & $1,04-1,69$ & 0,019 & 1,43 & $1,12-1,82$ & 0,004 \\
\hline $\begin{array}{l}\text { Possuir dois/um /nenhum } \\
\text { Bloco II }\end{array}$ & 1 & & & 1 & & \\
\hline \multicolumn{7}{|l|}{ Idade (anos) } \\
\hline $10-19$ & 1 & & & 1 & & \\
\hline $20-29$ & 1,79 & $1,36-2,36$ & 0,0001 & 1,40 & $1,03-1,90$ & 0,031 \\
\hline$\geq 30$ & 2,62 & $2,00-3,43$ & 0,0001 & 1,86 & $1,35-2,56$ & 0,0001 \\
\hline \multicolumn{7}{|l|}{$N^{\circ}$ de gestações } \\
\hline Nulíparas & 1 & & & 1 & & \\
\hline $1-2$ & 1,63 & $1,22-2,18$ & 0,001 & 1,37 & $1,00-1,87$ & 0,049 \\
\hline 3 ou mais & 2,42 & $1,82-3,21$ & 0,0001 & 1,70 & $1,22-2,38$ & 0,002 \\
\hline
\end{tabular}

Nota: "Televisão, geladeira, fogão e rádio; aAjustado por: situação geográfica do domicílio, bens de consumo, esgoto e lixo. 'Ajustado por: anemia e raça.

IC95\%: Intervalo de Confiança; RP: Razão de Prevalência.

mais representativo da transição nutricional no Estado de Pernambuco. Como exercício preditivo, estima-se que o déficit estatural de menores de cinco anos deve ser corrigido, alcançando o nível de controle da Desnutrição Energético Proteica $(D E P)^{16}$, enquanto a estimativa do excesso de peso em mulheres em idade fértil, se projetada a partir das tendências observadas no período 1997/2006, pode alcançar percentuais superiores a $50 \%$, tal como ocorre nos Estados Unidos e no Canadá2. Seria, portanto, um cenário epidemiológico que praticamente reflete a situação dos países desenvolvidos.

Na avaliação temporal e geográfica dessas tendências, algumas observações fundamentais devem ser destacadas. Revela-se que a maior elevação $(45,0 \%)$ da prevalência de excesso de peso ocorreu no meio rural, enquanto o aumento per- 
centual mais modesto verificou-se no interior urbano: apenas 6,8\%. Entre as duas tendências, portanto, o diferencial de ritmos foi de cinco vezes. Por sua importância quantitativa e, sobretudo, pela natureza qualitativa da mudança, ocorrendo no espaço territorial mais discriminado pelas restrições da pobreza, essas características são primordiais para a compreensão da transição nutricional que está ocorrendo no Estado, no Nordeste, no Brasil e, em escala mais moderada, na maioria dos países em desenvolvimento 2,6,17.

Ao lado das diferenças espaciais e, logicamente, com um componente explicativo mais relevante do processo de mudanças, destacam-se as observações de que, em todas as categorias referenciadas na classificação das variáveis exploratórias utilizadas nas análises, a ocorrência do excesso de peso aumentou entre 1997 e 2006. Trata-se, portanto, de uma tendência geral, embora implicando evidentes diferenças em função do grupo de variáveis consideradas. Concretamente, fica evidente que as diferenças geográficas registradas entre uma elevação mínima de 6,8\% no interior urbano, com melhores condições de vida, e o interior rural, de modo geral bem mais pobre, representam o efeito combinado de tendências interativas que se manifestam, em nível preliminar, nas análises bivariadas. Ou seja, a constatação inesperada de que, dentro de cada categoria de análise (renda, escolaridade, condições de saneamento, regime de ocupação das moradias, acesso a bens de consumo, por exemplo) foram as condições mais adversas que mais resultaram em aumento na prevalência do excesso de peso. Assim, as respostas estão se dando de fato, nas situações em que antes prevaleceriam os fatores de risco para os déficits nutricionais. Esse cenário é historicamente recente, atuando de forma detectável na metade da década de 1990, quando se produz o processo mais ativo da transição nutricional no Brasil. Em outra vertente, a explicação se estenderia ao plano biológico, assinalando as elevações de prevalência nas mulheres adolescentes e nas nulíparas, tendo como semelhança biológica a idade mais baixa.
Considerando simultaneamente fatores socioeconômicos, ambientais e biológicos, identificados nas análises bi e multivariados, parece bem evidente que as mudanças marcantes e até inesperadas demonstram a importância quali e quantitativa das rápidas transformações no cenário epidemiológico, caracterizando o processo de transição. A situação do excesso de peso em mulheres em idade reprodutiva no Estado de Pernambuco apenas acentua as mudanças que, num ritmo menos intenso, estão ocorrendo no Brasil e em grande parte dos países em desenvolvimento ${ }^{2,6}$. É uma mudança histórica e mais que conjuntural, envolvendo aspectos econômicos, políticos, culturais, éticos e ecológicos, constituindo o que se pode chamar de desenvolvimento humano ${ }^{1}$. Isso implica reconhecer as relações de desigualdade na produção e no uso de bens e serviços, no exercício de direitos e deveres, na rede de proteção social com seus vários componentes, na transição demográfica que altera a composição familiar e populacional, na consolidação da consciência coletiva sobre fatores de risco e de proteção, estabelecendo uma nova configuração do processo saúde/doença em nível coletivo. No caso do Brasil e da América Latina, esses aspectos já foram abordados por Batista Filho ${ }^{17}$, Monteiro \& Conde $^{18}$ e Kac \& Velásquez-Meléndez ${ }^{19}$.

A redução do ritmo de crescimento da epidemia do excesso de peso, notadamente em mulheres, já se faz há mais de duas décadas nos países nórdicos, há uma década nos Estados no Sul do Brasil e agora na Região Metropolitana do Recife (RMR), onde se concentram mais de 40\% de toda a população do Estado. Em outras palavras: seria a reprodução de uma tendência iniciada há algumas décadas no Norte da Europa Ocidental, refletindo-se no Sul do Brasil e que, na primeira década do século 21, começa a aparecer no Estado de Pernambuco.

Não sendo um comportamento peculiar ao Estado, possivelmente já caberia a interpretação de que, apesar das tendências de aumento na prevalência do excesso de peso, começa a se vislumbrar a perspectiva de uma nova mudança 
temporal, geográfica, social e biológica dos indicadores da situação nutricional das mulheres em idade fértil. Essa visão prospectiva parece apontar uma acentuada redução no ritmo de expansão da prevalência do excesso de peso na Região Metropolitana do Recife e, como lógica complementar, na observação de que, nas categorias de condições socioeconômicas e microambientais mais favoráveis, já se evidencia uma clara desaceleração do problema. A análise das tendências observadas neste estudo, assim como, no plano de validação externa, a literatura nacional ${ }^{17-19} \mathrm{e}$ a internacional, possibilitam essa predição.

Conclusivamente, é oportuno ponderar que as grandes mudanças, como as que caracterizam a transição nutricional, trazem em si uma carga considerável de imprevisibilidade. Dessa forma, a própria concordância eventual com outros estudos, outros autores e, sobretudo, outros espaços geográficos e outros momentos históricos não representam painéis preditivos para garantir observações mais consistentes. É surpreendente, convém repetir, que o excesso de peso corporal, em menos de um século, tenha se convertido em evento epidêmico de caráter grave nos tempos atuais. E que, contrariando a lógica da experiência acumulada, tenha revelado como categoria mais exposta as pessoas que até recentemente eram mais vulneráveis à desnutrição energético-proteica, tanto na sua forma aguda quanto crônica.

Agora, o sobrepeso/obesidade passa ocorrer como parte de um conjunto de comorbidades de longa duração (DCNT), deslocando sua focalização de risco para as etnias socialmente mais desfavorecidas, como negros e pardos, ou outros agrupamentos aproximados pela condição de pobreza, como famílias do meio rural, estratos de renda mais baixa ou em condições inadequadas de saneamento da moradia e do meio ambiente.

A propósito, é oportuno considerar a pertinência e a relevância de um novo Inquérito, programado para 2015, a IV PESN, dentro dos mesmos objetivos e procedimentos metodológicos das pesquisas que serviram de suporte para o trabalho aqui relatado. Na mesma lógica, vale justificar a decisão dos Ministérios da Saúde e do Desenvolvimento Social, assegurando a realização, a cada cinco anos, de um inquérito representativo das condições de saúde e nutrição no país e suas macrorregiões. É uma estratégia ágil e competente para dispor de referenciais seguros e atualizados, diante de uma situação que está em permanente e rápida transformação.

\section{COLABORADORES}

M BATISTA FILHO e PIC LIRA participaram da elaboração dos projetos de estudo e dos instrumentos de coleta de dados, da supervisão dos trabalhos de campo, do levantamento bibliográfico, da análise estatística e da redação científica do trabalho. EC COSTA participou do levantamento bibliográfico, do processamento, da análise estatística e da redação do artigo. JS OLIVEIRA, RCM EGITO e FCLP TAVARES participaram da elaboração dos instrumentos de campo, da coleta e tabulação dos dados.

\section{REFERÊ NCIAS}

1. Batista Filho M, Batista LV. Transição alimentar/nutricional ou mutação antropológica? Cienc Cult. 2010; 62(4):26-30.

2. World Health Organization. Global status report on noncommunicable diseases 2010. Geneva: World Health Organization; 2011 [cited 2013 Nov 10]. Available from: <http://www.who.int/nmh/ publications/ncd_report2010/ en/index.html>.

3. World Health Organization. Global nutrition policy review: What does it take to scale up nutrition action? Geneva: World Health Organization; 2013 [cited 2013 Nov 5]. Available from: <http://www. who.int/nutrition/publications/policies/global_nut_ policyreview/en/>.

4. Schmidt MI, Duncan BB, Azevedo e Silva G, Menezes AM, Monteiro CA, Barreto SM, et al. Chronic noncommunicable diseases in Brazil: Burden and current challenges. Lancet. 2011; 377(9781):1949-61. doi: 10.1016/S0140-6736(11)60135-9

5. Brasil. Secretaria de Vigilância em Saúde. VIGITEL Brasil 2011: vigilância de fatores de risco e proteção para doenças crônicas por inquérito telefônico. Brasília: Ministério da Saúde; 2012. 
6. Instituto Brasileiro de Geografia e Estatística. Pesquisa de orçamentos familiares 2008-2009: antropometria e estado nutricional de crianças, adolescentes e adultos no Brasil. Rio de Janeiro: IBGE; 2010.

7. Vieira ACR, Sichieri R. Associação do status socioeconômico com obesidade. Physis. 2008; 18(3):415-26. doi: 10.1590/S0103-73312008000 300003

8. Wanderley EM, Ferreira VA. Obesidade: uma perspectiva plural. Cienc Saúde Colet. 2010; 15(1):185-94.

9. Gutierrez-Fisac JL, Regidor E, Banegas JRB, Artalejo FR. The size of obesity differences associated with educational level in Spain, 1987 and 1995/97. J Epidemiol Comm Health. 2002; 56(6):457-60.

10. Torrance GM, Hooper MD, Reeder BA. Trends in overweight and obesity among adults in Canada (1970-1992): Evidence from national surveys using measured height and weight. Int J Obes Relat Metab Disord. 2002; 26(6):797-804.

11. Monteiro CA, Moura EC, Conde WL, Popkin BM. Socioeconomic status and obesity in adult populations of developing countries: A review. Bull World Health Organ. 2004; 82(12):940-6.

12. World Health Organization. Iron deficiency anemia: Assessment, prevention and control: A guide for programme managers. Geneva: WHO; 2001.

13. World Health Organization. Physical status: The use and interpretation of anthropometry. Geneva: WHO; 1995. Technical Report Series, nº 854.
14. Brasil. Ministério da Saúde. Secretaria de Políticas de Saúde. Saúde da criança: acompanhamento do crescimento e desenvolvimento infantil. Brasília: Ministério da Saúde; 2002. Série Cadernos de Atenção Básica, nº 11.

15. De Onis M, Onyango AW, Borghi E, Siyam A, Nishida C, Siekmann J. Development of a WHO growth reference for school-aged children and adolescents. Bull World Health Organ. 2007; 85(9):660-7.

16. Figueiroa JN, Alves JG, Lira PI, Batista Filho M. Evolução intergeracional da estatura no Estado de Pernambuco, entre 1945 e 2006: 2- aspectos analíticos. Cad Saúde Pública. 2012; 28(8):1468-78.

17. Batista Filho M, Souza Al, Miglioli TC, Santos MC. Anemia e obesidade: um paradoxo da transição nutricional brasileira. Cad Saúde Pública. 2008; 24(Suppl 2):S247-57.

18. Monteiro CA, Conde WL. A tendência secular da obesidade segundo estratos sociais: nordeste e sudeste do Brasil, 1975-1989-1997. Arq Bras Endocrinol Metabol. 1999; 43(3):186-94.

19. Kac G, Velásquez-Meléndez G. A transição nutricional e a epidemiologia da obesidade na América Latina. Cad Saúde Pública. 2003; 19(1): 4-5. 